Abanico Veterinario. Enero-Diciembre 2021; 11:1-7. http://dx.doi.org/10.21929/abavet2021.1

Caso Clínico. Recibido: 29/08/2020. Aceptado: 03/12/2020. Publicado: 04/01/2021. Clave: 2020-51.

\title{
Infección secundaria por Leucobacter chromiiresistens en un perro con schwannoma axillar malingo tipo III
}

Secondary infection by Leucobacter chromiiresistens in a dog with malignant axillary schwannoma type III

\section{Rivero-Perez Nallely ${ }^{1 \mathrm{ID}}$, Ocampo-López Juan ${ }^{1 \mathrm{ID}}$, Vera-Borja Gerardo', Hori-Oshima Sawako ${ }^{\text {ID }}$, Valladares-Carranza Benjamín ${ }^{3}$ ID, Rosenfeld-Miranda Carla ${ }^{4}$ ID, Zaragoza-Bastida Adrian*1 ID}

${ }^{1}$ Área Académica de Medicina Veterinaria y Zootecnia, Instituto de Ciencias Agropecuaria, Universidad Autónoma del Estado de Hidalgo. México. ${ }^{2}$ Instituto de Investigaciones en Ciencias Veterinarias, Universidad Autónoma de Baja California, Mexicali, Baja California, México. ${ }^{3}$ Facultad de Medicina Veterinaria y Zootecnia, Universidad Autónoma del Estado de México. México. ${ }^{4}$ Facultad de Ciencias Veterinarias, Universidad Austral de Chile, Valdivia, Chile. *Autor para correspondencia: Zaragoza-Bastida Adrian. Rancho Universitario, Av. Universidad Km 1, Ex-Hda. de Aquetzalpa, C.P. 43600 Tulancingo, Hidalgo, México. nallely_rivero@uaeh.edu.mx, jocampo@uaeh.edu.mx, shori@uabc.edu.mx, benvac2004@yahoo.com.mx, adrian_zaragoza@uaeh.edu.mx

\section{RESUMEN}

El presente reporte describe un schwannoma tipo III encontrado en un macho SharPei de 12 años, la neoplasia fue retirada y analizada por histopatología e inmunohistoquímica. El schwannoma fue clasificado con alta malignidad y con metástasis al linfonodo axilar. Con técnicas de rutina y moleculares se identificó una septicemia bacteriana secundaria. El diagnostico inmunohistoquímico confirmó el diagnóstico presuntivo de schwannoma, así como su grado de malignidad. Adicionalmente se aisló e identificó Leucobacter chromiiresistens, especie de la cual a la fecha no hay reportes como patógeno de animales o humanos.

Palabras clave: Schwannoma, SharPei, Leucobacter chromiiresistens.

\section{ABSTRACT}

The present report describes a schwannoma type III found in a 12-year-old male SharPei dog, this neoplasm was excised and analyzed using histopathological and immunohistochemistry examinations. This schwannoma was classified as high malignancy and presented metastasis to the axillary node. A secondary bacterial septicemia was identified with routine and molecular techniques. The Inmunohistochemical diagnosis of the mass, corroborated the presumptive diagnosis, which suggested a Schwannoma as well as its malignancy degree. In addition, Leucobacter chromiiresistens was isolated, a specie of which to date there are no reports as a pathogen in animals or humans.

Keywords: Schwannoma, SharPei, Leucobacter chromiiresistens.

\section{INTRODUCCIÓN}

Los tumores cutáneos de origen neurológico son poco frecuentes en animales domésticos, sin embargo, es probable que sean poco diagnosticados, debido a la similitud histológica que presenta respecto a otros tumores cutáneos más frecuentes. El nombre de tumor de la vaina del nervio periférico (TNP) es un término amplio propuesto para incluir aquellos tumores que surgen de los nervios periféricos (neurofibromas/ 
neurofibrosarcomas/ neurinomas, neurilemmomas y schwannomas); sin embargo, debido a que la mayoría de los tumores están constituidos por células de Schwann, el término schwannoma es apropiado para la mayoría de estos tumores (Maxie et al., 2016).

El schwannoma (neurilemoma) es un tumor de nervios mielinizados que se origina en la vaina de los neurolemocitos (células de Schwann), que puede tener una presentación benigna o maligna. Existen reportes de casos de neurilemomas en animales, incluidos perros, la incidencia aproximada de estos tumores en perros es de 3.8 en 100,000 animales y se observan con mayor frecuencia en nervios craneales, nervios del plexo braquial y en nervios de la séptima cervical, causando compresión de la médula espinal (Ramírez et al., 2000; Baka et al., 2017). El schwannoma en perros ha sido reportado en la pared uveal, orbital, perianal y torácica entre otros, sin embargo normalmente son subcutáneos, la localización más común de los neurilemomas cutáneos en perros son las extremidades y el dorso (Maxie et al., 2016; Gaitero et al., 2008). Los animales con tumores son susceptibles a diversas afecciones metabólicas y fisiológicas, así como infecciones secundarias incluyendo aquellas de origen bacteriano (Azap et al., 2012). El presente reporte describe un schwannoma tipo III en un perro con una infección secundaria, asociada a Leucobacter chromiiresistens.

\section{Historia del caso}

Un perro Shar-pei, macho de 12 años de edad, visitó el Hospital Veterinario de la Universidad Autónomas del Estado de Hidalgo, para la evaluación de un crecimiento anormal en la región axilar derecha. El examen físico general reveló, enfermedad periodontal grado II, condición corporal $4 / 9$ y la presencia de una masa subcutánea de tejido recién formada, localizada en la región axilar derecha. La masa $(15 \mathrm{~cm}$ de largo, 5 $\mathrm{cm}$ de ancho, $3 \mathrm{~cm}$ de profundidad) era de aspecto multilobulado, no dolorosa, firme, ligeramente desplazable y con alopecia en la zona.

Se realizaron un hemograma y un perfil bioquímico, no se reportaron anormalidades en el hemograma, pero el perfil bioquímico mostró hiperproteinemia marginal asociada a reacción inflamatoria. Se colectó una muestra de sangre por medio del sistema BD Vacutainer® en un tubo con heparina para hemocultivo, de acuerdo con la metodología descrita en el Manual de Productos y Procedimientos de Laboratorio (Zimbro et al., 2009). Se realizó una nodulectomía una semana después del examen físico, la cual incluyó una incisión en la región axilar del área del plexo braquial. Se observó la formación de un nuevo tejido altamente vascularizado, poco delimitado, no encapsulado y asimétrico, e involucraba el nervio musculocutáneo y posiblemente el nervio radial. Debido a que se observó que la neoplasia se infiltró significativamente en el tejido muscular subyacente, se realizó una resección parcial del músculo pectoral profundo. Aunado a esto se observó un linfonodo regional agrandado y firme. El tumor y linfonodo regional fueron extirpados y fijados en formalina al $4 \%$ para su posterior estudio histopatológico e inmunohistoquímico (Prophet et al., 1995). 
En la masa tumoral se encontraron numerosas células neoplásicas en forma de huso, dispuestas en haces cortos entrelazados (cuerpos de Verocay), sostenidas y delimitadas por estroma fibrovascular (Figura 1, A). En otras partes se observaron extensas áreas de material eosinofílico granular y con pocas células fusiformes (Patrón histológico Antoni A y Antoni B) (Musha et al., 2018; Salazar et al., 2016).

Las células fueron fusiformes, con bordes celulares poco definidos, pérdida moderada del citoplasma, núcleo central ovalado con bordes filosos, cromatina granular gruesa y moderada anisocitosis, anisocariosis, y cariomegalia (Figura 1, B), así mismo fueron contadas cuarenta y tres figuras mitóticas en $2.37 \mathrm{~mm}^{2}$ en la periferia del tumor, área con la mayor actividad mitótica. Se observaron áreas extensas de necrosis coagulativa, hemorragia y grupos de linfocitos y neutrófilos entre las células. Los vasos linfáticos se observaron distendidos por grupos de células neoplásicas fusiformes. Estos hallazgos fueron indicativos de un neurilemoma maligno (schwannoma) grado III (alto grado de malignidad) (Ahmadi et al., 2012; Sharif et al., 2017).
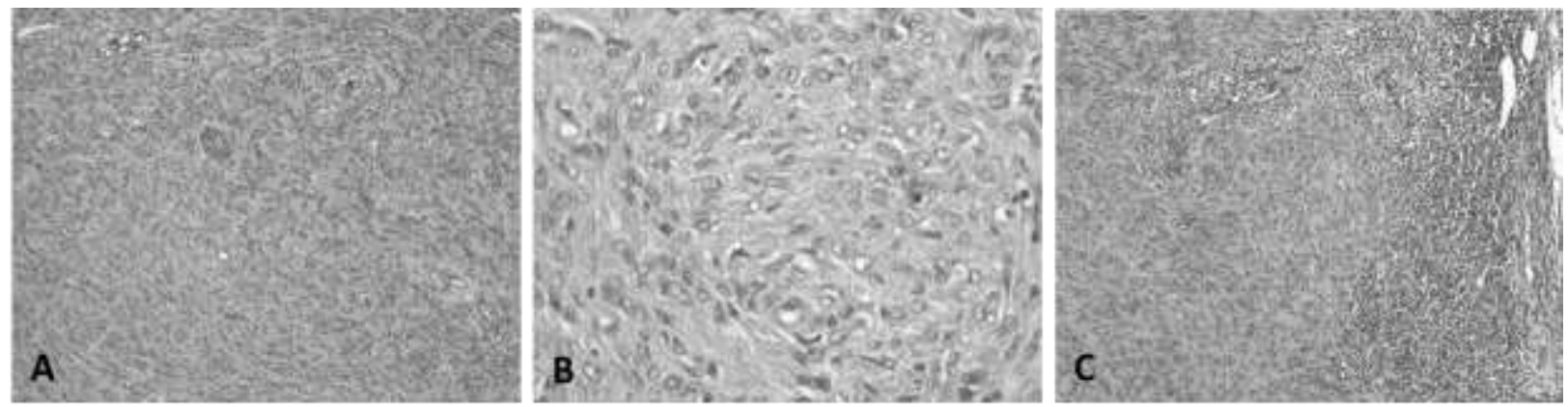

Figura 1: Neurilemmoma. Canino, Shar-pei, macho, 4 años. H-E. A. 100X.

Apariencia del tumor: A. Las células neoplásicas, en forma de huso, están dispuestas en haces cortos (cuerpos de Verocay), orientadas en los tres planos del espacio y sostenidad por estroma fibrovascular, relativamente escaso. B. 400X. Detalles de la celularidad tumoral. Las células son fusiformes, con bordes poco definidos, núcleo central ovalado, con cromatina granular gruesa y moderada anisocitosis, anisocariosis y cariomegalia. C. 100X. Metástasis del linfonodo regional: El tejido neoplásico ocupa la corteza y parcialmente la médula del linfonodo.

En el linfonodo regional, hubo una reducción de las células linfoides, observandose $60 \%$ de linfoblastos, $15 \%$ de los linfocitos maduros, $15 \%$ de células plasmáticas y $10 \%$ de macrófagos. Los senos medulares revelaron densos grupos de células fusiformes que reemplazaron una gran parte del parénquima del linfonodo, con una corteza muy delgada y sin la presencia característica de linfonodos (Figura 1, B). Estos hallazgos corresponden con una permeación linfática y metástasis a los linfonodos (Ahmadi et al., 2012). 
Para determinar el origen tisular de las células, se realizó un estudio inmunohistoquímico indirecto, a través de la técnica de estreptavidina-biotina-peroxidasa con anticuerpos policlonales, que dieron positivo para la proteína ácida fibrilar glial (Figura 2, A), proteína S-100 (Figura 2, B), y vimentina (Figura 2, C).
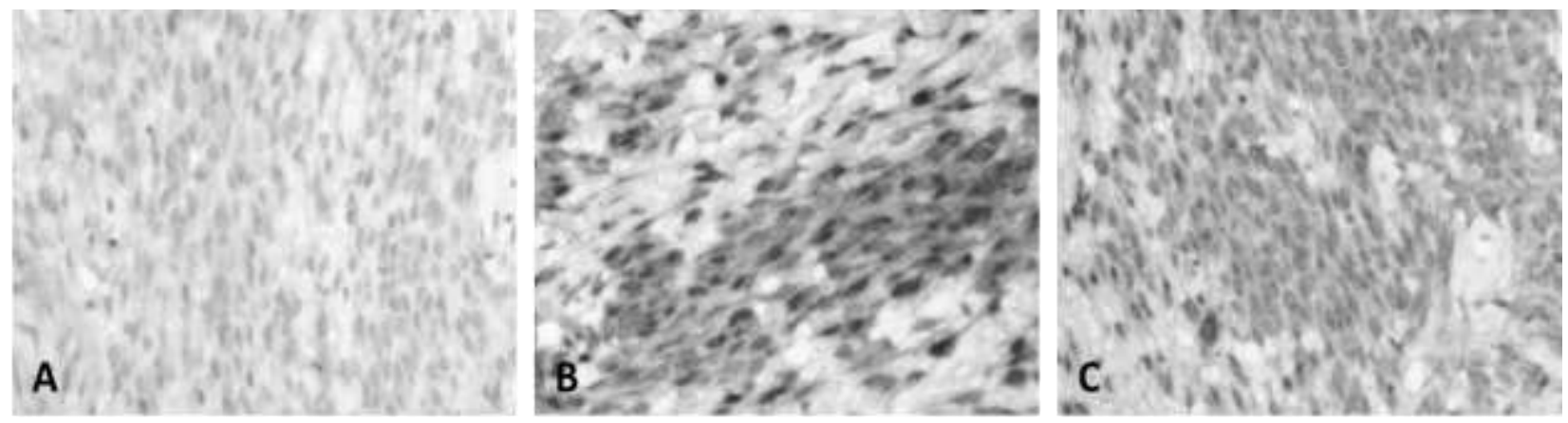

Figura 2: Neurilemoma. Canino, Shar-pei, macho, 4 años. Inmunomarcaje con HRP. 400X. A. Proteína ácida fibrilar glial, en un patrón discreto pero uniforme en la mayoría de las células neoplásicas. B. Proteína S-100, marcado muy notorio, en grupos de células tumorales C. Vimentina, marcaje uniforme en grupos celulares del neurilemoma.

En el procesamiento de la muestra a través de técnicas bacteriológicas, se observó el crecimiento de colonias pigmentadas de color amarillo, con presencia de bacilos Gram positivos. La cepa aislada se identificó por medio de la amplificación, secuenciación y comparación con el gen 16S rRNA (Zaragoza et al., 2017). Los resultados del análisis molecular mostraron que la cepa tuvo una similitud del $99,4 \%$ con Leucobacter chromiiresistens (Tabla 1 y Figura 3).

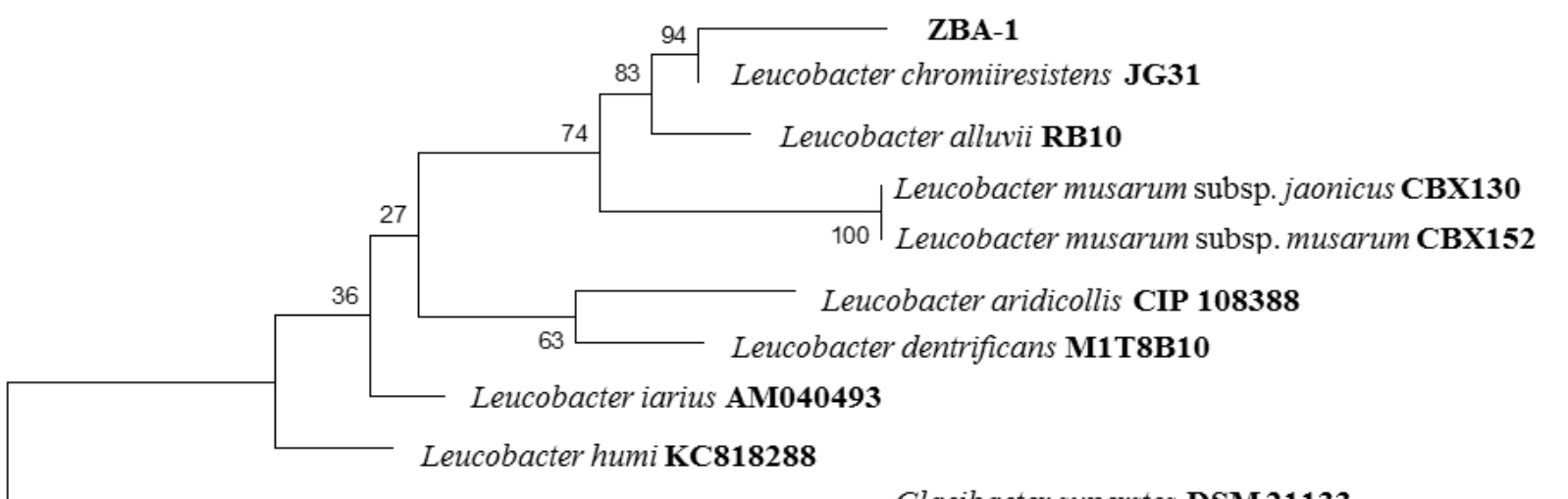

Glacibacter superstes DSM21133

0.005

Figura 3. Árbol filogenético construido a través de la comparación de la secuencia del gen rRNA $16 S$ de la cepa aislada y las cepas de referencia. 


\section{DISCUSIÓN}

Los schwannomas pueden tener diferentes localizaciones y presentaciones, en este estudio se reporta un schwannoma tipo III con alto grado de malignidad y con metástasis al linfonodo axilar, en un macho Shar-Pei de 12 años. Los schwannomas no son comunes en perros, sin embargo en un estudio realizado por Gaitero et al., en 2008 se reportaron dos casos de TNP caninos, en la región axilar derecha, ambos en perros de raza mestiza, uno de los tumores tuvo una dimensión de $3 \times 2 \mathrm{~cm}$, similar al schwannoma reportado en el presente caso $(5 \times 3 \mathrm{~cm})$, dichos autores mencionan que no existe predisposición racial para este tipo de tumores, así mismo se menciona que los animales mas afectados son perros geriatras, como ocurrió en el presente caso. En la literatura se reporta que aproximadamente el $42 \%$ de los perros diagnosticados con schwannomas han sido eutanasiados, situación similar a la del presente caso (Jones et al., 1975), después del procedimiento quirúrgico, el paciente fue enviado a casa con tratamiento analgésico, el cual incluía meloxicam $0.1 \mathrm{mg} / \mathrm{kg}$, tramadol $3 \mathrm{mg} / \mathrm{kg}$, cefalexina $30 \mathrm{mg} / \mathrm{kg}$, y omeprazol $1 \mathrm{mg} / \mathrm{kg}$, se recomendó reposo y el uso de collar Isabelino. Sin embargo, siete dias despúes de la cirugía, el paciente presentó debilidad generalizada, membranas mucosas pálidas, taquicardia, pulso débil, y pérdida de $2 \mathrm{~kg}$ de peso. Debido al gradual deterioro del paciente, los miembros de la familia decidieron optar por el procedimiento de eutanasia, sin otorgar la autorización para la necropsia. Los resultados del análisis molecular de la bacteria determinaron que la cepa tuvo una similitud del $99.4 \%$ con Leucobacter chromiiresistens, un bacilo irregular Gram positivo, no móvil, de coloración amarilla, a la fecha no existen reportes de infección en humanos o animales a causa de estas especies (Sturm et al., 2011).

Table 1. Comparación del gen rRNA 16S de la cepa aislada con las depositadas en el GenBank, usando BLAST y EzTaxon

\begin{tabular}{|c|c|c|c|c|}
\hline Cepa & $\begin{array}{c}\text { Tamaño } \\
(\mathrm{Pb})\end{array}$ & Especies similares & Similitud GenBank (\%) & Similitud Eztaxon (\%) \\
\hline \multirow{6}{*}{ ZBA-1 } & \multirow{6}{*}{1400} & Leucobacter chromiiresistens & 99 & 99.4 \\
\hline & & Leucobacter alluvii & 98 & 98.7 \\
\hline & & Leucobacter musarum subsp. japonicus & 98 & 98.0 \\
\hline & & Leucobacter musarum subsp. musarum & 98 & 98.0 \\
\hline & & Leucobacter humi & 97 & 97.7 \\
\hline & & Leucobacter iarius & 97 & 97.4 \\
\hline
\end{tabular}

De acuerdo con la literatura, Escherichia coli, Klebsiella pneumoniae, Pseudomonas aeruginosa, Staphylococcus aureus, Streptococcus pneumoniae, Streptococcus pyogenes, y Bacteroides spp comúnmente se asocian a infecciones secundarias en pacientes con cáncer (Lopardo et al., 2014). El género Leucobacter no es común en infecciones secundarias en pacientes con cáncer, sin embargo existen reportes de 
infecciones a causa de éste género, principalmente en pacientes con cáncer terminal (Adderson et al., 2008). Las lesiones causadas debido a la infección secundaria por Leucobacter chromiiresistens no fueron identificadas ya que la necropsia no fue autorizada.

\section{CONCLUSIÓN}

El diagnóstico inmunohistoquímico de la masa tumoral, confirmó el diagnóstico presuntivo, que sugería un schwannoma así, como el grado de malignidad que presentaba. Adicionalmente se aisló Leucobacter chromiiresistens, una especie de la cual a la fecha no existen reportes de que sea un agente patógeno para humanos o animales.

\section{AGRADECIMIENTOS}

Los autores desean agradecer el apoyo de la Secretaría de Investigación de la Universidad Autónoma del Estado de Hidalgo y un agradecimiento especial al Dr. Julio C. Osorio Baños+ por todas sus contribuciones a este reporte de caso.

\section{REFERENCIAS}

ADDERSON EE, Boudreaux JW, Hayden RT. 2008. Infections Caused by Coryneform Bacteria in Pediatric Oncology Patients. The Pediatric Infectious Disease Journal. 27(2):136-41. https://doi.org/10.1097/INF.0b013e31814fab12

AHMADI N, Oryan A, Ghane M, Daneshbod Y. 2012. Cutaneous schwannoma in a cow. Brazilian Journal of Veterinary Pathology. 5(2):81-85. http://bjvp.org.br/wpcontent/uploads/2015/07/DOWNLOAD-FULL-ARTICLE-18-

20881_2012_8_18_23_56.pdf

AZAP A, Bozkurt GY, Yüksel MK, Kutlu H, Topçuoğlu P, Aypak A. 2012. Secondary infections in cancer patients with febrile neutropenia. Turkish Journal of Hematology. 29 (3):254-258. https://www.journalagent.com/tjh/pdfs/TJH_29_3_254_258.pdf

BAKA O, Psalla D, Soubasis N, Polizopoulou Z. 2017. Cranial vena cava syndrome in a Dog with a brachial plexus malignant peripheral nerve sheath tumour. Australian Veterinary Practitioner. 47(4):113-116.

https://www.researchgate.net/publication/330412296_Cranial_Vena_Cava_syndrome_in a_dog_with_a_brachial_plexus_malignant_peripheral_nerve_sheath_tumour

GAITERO L, Anor S, Fondevila D, Pumarola M. 2008. Canine cutaneous spindle cell tumours with features of peripheral nerve sheath tumours: a histopathological and immunohistochemical study. Journal of Comparative Pathology. 139(1):16-23. https://doi.org/10.1016/j.jcpa.2008.03.003

JONES BR, Williams OJ. 1975. Malignant schwannoma of the brachial plexus in a dog. Australian Veterinary Journal. 51(1):40-42. https://doi.org/10.1111/j.17510813.1975.tb14496.x 
LOPARDO HA. 2014. Infecciones por bacterias poco comunes y oncogénesis bacteriana. Revista Argentina de Microbiología. 46(1):1-6. https://doi.org/10.1016/S03257541(14)70066-5

MAXIE G. 2016. Nervous system en: Jubb, Kennedy \& Palmer's. Pathology of Domestic Animals. St. Louis, Missouri EEUU: Elsevier Health Sciences. Pp. 404-405. ISBN: 978-07020-5317-7

MUSHA A, Ogawa M, Yokoo S. 2018. Granular cell tumors of the tongue: fibroma or schwannoma. Head \& Face Medicine. 14(1):1-7. https://doi.org/10.1186/s13005-0170158-9.

PROPHET EB, Mills B. Métodos Histotecnológicos. 1995. Instituto de Patología de las Fuerzas Armadas de los Estados Unidos de América (AFIP). Washington, D.C. United State of America.

RAMÍREZ DM, Calzada LA, Colín FR. 2000. Schwannoma en la región pélvica de un perro de raza Doberman. Veterinaria México OA. 31(2):169-72. https://www.redalyc.org/pdf/423/42331215.pdf

SALAZAR MF, Tena Suck ML, Rembao Bojorquez D, Salinas Lara C. 2016. Intraventricular Neurilemmoma (Schwannoma): Shall GFAP Immunostaining Be Regarded as a Histogenetical Tag or as a Mere Histomimetical Trait. Case Reports in Pathology. 2016:6-12. http://dx.doi.org/10.1155/2016/2494175

SHARIF M, Mohamed A, Reinacher M. 2017 Malignant renal schwannoma in a cat. Open Veterinary Journal. 7(3):214-20. http://dx.doi.org/10.4314/ovj.v7i3.3

STURM G, Jacobs J, Spröer C, Schumann P, Gescher J. 2011. Leucobacter chromiiresistens sp. nov., a chromate-resistant strain. International Journal of Systematic and Evolutionary Microbiology. 61(4):956-60. https://doi.org/10.1099/ijs.0.022780-0

ZARAGOZA BA, Rivero PN, Valladares CB, Isaac-OK, Moreno PP, Sandoval TH. 2017. Molecular Identification of Mycobacterium Species of Public Health and Veterinary Importance from Cattle in the South State of México. Canadian Journal of Infectious Diseases and Medical Microbiology. 2017:1-7. https://doi.org/10.1155/2017/6094587

ZIMBRO MB, Power DA, Miller SM, Wilson GE, Johnson JA. 2009. Manual of Microbiological Culture Media. Second Edition. Maryland United State of America. ISBN 0-9727207-1-5. https://www.trios.cz/wp-content/uploads/sites/149/2016/08/DIFCO-ABBL-MANUAL-2.pdf 